\title{
Inclusion of physical therapy services on a short term mobile medical mission team to Nicaragua: a qualitative description study of team members' observations and recommendations for improvement
}

\author{
Douglas Steele ${ }^{\mathrm{a}}$ and Candice Beitman ${ }^{\mathrm{b}}$ \\ ${ }^{a}$ DHS, DPT, MHS, PA-C, PT, CPT, Physican Assistant: UF Health Department of Orthopedic Surgery and \\ Rehabiliation \\ ${ }^{\mathrm{b}}$ OT EdD, Retired Associate Professor, School of Occupational Therapy, University of Indianapolis
}

\section{Abstract}

Context: Access to physical therapy (PT) services is sorely limited in many developing countries due to the constraints of poverty and regional insufficiency in the number of PT providers. Opportunities exist for physical therapists to participate in short term mobile medical mission (STMMM) efforts to provide PT services in many of those regions. However, there is a relative dearth of research regarding the utility of including PT services in STMMM endeavors.

Objective: The aim of this study was to ascertain the impressions and recommendations of medical mission team members regarding the inclusion of PT services during a STMMM trip to underserved areas of Nicaragua.

Methods: This was a qualitative description study conducted in 2014. Data were derived from interviews with STMMM participants and analyzed using conventional content analysis as described by Hsieh and Shannon.

Results: Emergent themes included evidence of: (1) patient need for and benefit from PT services; (2) team member need for physical therapy services for trip related disorders; and (3) a high prevalence of patient orthopedic disorders related to strenuous activities of daily living. There were also a number of reported limitations in the delivery of PT services including: (1) language barriers; (2) lack of medical provider familiarity with PT capability; and (3) limited means of providing follow-up care. Respondent recommendations included: (1) addition of more translators; (2) inclusion of additional treatment modalities; (3) utilization of Nicaraguan therapists or health care workers to facilitate patient follow-up; and (4) the provision of a comprehensive screening program to improve identification of patients in need of PT services.

Conclusion: This study contributes to the understanding of corollaries associated with the addition of PT services to a STMMM and provides recommendations to improve

Nov 2015. Christian Journal for Global Health, 2(2):39-51. 
PT services in that context. Additional research is needed to evaluate the soundness of participant responses and whether this information is transferable to STMMM efforts in other communities. Further research is also needed to establish best PT practices in the STMMM context.

\section{Introduction}

The need to improve global health care is agonizingly evident but that mission is fraught with barriers. Peters et al. found that people in impoverished countries tend to have less access to health services than those in more prosperous countries and that the poverty-stricken within countries tend to have less access to healthcare as well. ${ }^{1}$ Access to health care is also negatively affected by a low ratio of healthcare providers to population in many parts of the world. In 2013, the WHO Global Health Observatory revealed that 83 primarily low income countries located in Central Africa, Southern Asia, Indonesia, and Central America fell below a density threshold of 22.8 skilled health professionals per 10,000 in population. $^{2}$

This deleterious synergy of poverty and regional inadequacy of health care providers is a major factor in limiting access to rehabilitation services as well. A 2011World Report on disability from the WHO estimated that nearly one billion people worldwide, mostly in low and middle income countries, experience some form of disability that requires rehabilitation services. ${ }^{3}$ Unfortunately, these are also the countries with the fewest number of rehabilitation professionals. Liao et al. evaluated member countries of the World Confederation for Physical Therapy from Europe, Oceania, North America, Latin America, Asia, and Africa in the 1990s and found that the median number of physical therapists was 167 per million people. ${ }^{4}$ A 2011 study by Gupta et al. revealed similar findings of low and middle income countries having the greatest need for rehabilitation services and the least availability of skilled rehabilitation professionals. ${ }^{5}$
Nicaragua is a superlative example of a low income country in which there is a great need for rehabilitation services and a relative dearth of physical therapists, particularly in rural areas. ${ }^{6,7}$ In 2003, Cano, Coordinator of Rehabilitation for the National Technological Institute in Nicaragua, estimated the rate of disability in Nicaragua at close to $15 \%$ of the population. ${ }^{8}$

The number of people with disorders needing physical therapy (PT) intervention in Nicaragua coupled with the barriers of poverty and the limited numbers of rehabilitation professionals available to provide care makes expediting the availability and accessibility of PT services a paramount health care issue.

There are a number of ministry institutions that include PT in short term mobile medical mission (STMMM) efforts in Nicaragua and other countries with limited access to rehabilitation services. ${ }^{9-13}$ However, there is a relative dearth of research available regarding the functionality and utility of including PT services in STMMM endeavors.

In 2009, Bajkiewicz categorized the three main services of a short term medical mission described in the literature as relief care, during which emergency-response services are provided post disaster; surgical-dental teams, which provide surgical and dental procedures in underdeveloped regions, and mobile clinics, during which mobile health clinics are set up in outlying villages where medical care is limited or nonexistent. ${ }^{14}$ The mobile clinics Bajkiewicz described have the essentially the same structure and purpose as the STMMM referred to in this research.

There are a limited number of publications that discuss the role of physical therapists in what Bajkiewicz categorized as relief care. ${ }^{14}$ Ketter 
reported a physical therapist's account of his role and inter-professional dynamics while assisting a 40-member emergency medical relief team in Nicaragua post Hurricane Mitch in 1998. ${ }^{15}$ The physical therapist reported that he was initially assigned to assist in the dental clinic, but when the medical providers learned there was a physical therapist available, they requested consults for patients with orthopedic conditions, open wounds, and mobility limitations. Team members also requested treatment by the physical therapist for various musculoskeletal complaints.

Nixon et al. examined the role of physical therapists in STMMM for disaster relief in Haiti after a 7.0 earthquake struck Port-au-Prince in January of 2010. ${ }^{16}$ Rehabilitation needs for people with spinal-cord injuries, amputations, head injuries, and other forms of disablement were reported. Faanes et al. described the experiences of physical therapists from United States, Canada, Africa, and the United Kingdom who participated in another relief effort after the 2010 earthquake in Haiti. ${ }^{17}$ Common experiential elements reported included: (1) emotional responses of fear, uncertainty, and gratification; (2) challenges of financial strain, language barriers, coping with devastation, and a wider scope of practice; (3) realization that education of patients, patient's families, and volunteers was critical to success of the relief effort; and (4) a greater sense of responsibility to help people during crisis. Participants in the study by Faanes and colleagues also described their roles as clinicians, educators, and consultants as adding to the quality of care patients received following the earthquake. ${ }^{17}$

Currently available literature provides tangential insight at best into the utility of including PT services in non-emergent STMMM. In lieu of the paucity of contextual research, the purpose of this study was to ascertain the impressions and recommendations of medical mission team members regarding the inclusion of PT services during a STMMM trip to areas of Nicaragua with limited access to rehabilitation professionals.
Determining and evaluating the benefits, limitations and recommended improvements for a mobile medical clinic that includes PT services will enable better planning and execution of future clinics that include rehabilitation professionals. They provide foundational insight for additional research to investigate the clinical efficacy of physical therapy services delivered in the STMMM context.

\section{Methods}

This study was approved by the University of Indianapolis IRB on August 8 ${ }^{\text {th }}, 2013$.

Qualitative description was used to address the research questions, "What are the observations of medical mission team members regarding the inclusion of PT services on a mobile medical mission team to Nicaragua, and what are their recommendations for improvement in the delivery of those services?" Sandelowski purports that qualitative description is the ideal research method to facilitate a straightforward depiction of phenomena which was the goal of this study. ${ }^{18}$ This approach facilitated the procurement of practical information that could improve planning and execution of PT services during future mission trips and enabled the discernment of operational and patient care issues that were in need of further research.

The STMMM from which this research originated took place in Apanas, Yankee, and Anita, three rural villages in the department of Jinotega, Nicaragua in cooperation with Mission Para Cristo. Mission Para Cristo is a Christian faith based ministry that works collaboratively with Nicaraguan community and church leaders to provide spiritual, medical, educational, and sustenance resources to impoverished communities within Nicaragua.

Nicaraguans living in rural settings have access to limited healthcare from the MINSA Health Department Outposts. ${ }^{19}$ However, there are no MINSA Outposts near the villages of Apanas, Yankee, and Anita. People living in those communities have difficulty accessing health services, especially more specialized services such 
as PT due to the scarcity of providers in the region, difficultly traveling to these sites, and inability to pay for private sector health services.

The inclusion criterion was mobile medical mission team members with US stateside phone service. The exclusion criterion was mobile medical mission team members with very limited or no interaction with the delivery of PT services during the mission trip as determined by question one of the post clinic interview. All individuals meeting the inclusion criterion and agreeing to participate in the study reviewed and signed an informed consent document. Each of the individuals who agreed to participate in this research was given a copy of the study interview questions during the informed consent process. (Appendix A)

Research participants included: (1) three physical therapists with aggregate expertise in orthopedics, neurology, gerontology, and pediatrics; (2) a broad range of medical professionals including a physician specializing in internal medicine, a nurse practitioner/dietician, a physician assistant /physical therapist, a pharmacist, and triage nurse; and (3) eleven nursing students that included both entry level and RN to BSN transition students.

Each clinic day, patients were triaged by a nurse with the assistance of nursing students and referred to medical providers first but on occasion directly to PT based on patient complaints. All patients receiving PT for whom synergistic medication was indicated were assessed by medical providers and screened for drug allergies, contraindications, and comorbidities prior to prescribing those medications. This interactive format facilitated consultations between the PT and other professionals on a regular basis.

A semi-structured phone interview was performed and digitally recorded within 14 days of the last clinic by the primary researcher to enhance dependability (the degree to which data and its analysis remain stable over time). ${ }^{20}$ Seventeen of the 19 individuals who agreed to participate were ultimately included in the study. One individual could not be reached by phone and one individual indicated she did not have the opportunity to observe or participate in PT in any significant way and was therefore excluded from the study.

The digital recordings were then transcribed verbatim by the primary researcher. Intermittent rechecks of the accuracy of transcription was performed periodically during the process. The data was de-identified to ensure confidentiality with methods recommended by McLellan et al. ${ }^{21}$

Upon completion of the de-identification process, data analysis of the transcripts was performed using qualitative content analysis. According to Hsieh-Fang and Shannon, conventional content analysis is a good option when: (1) the study aim is description of a phenomenon; (2) there is limited research on the topic; (3) open ended inquiry is used to acquire data; (4) and when direct information from participants that has not been influenced by preconceived categorization or theory is desired. ${ }^{22}$ These were all features of our research project; therefore, conventional content analysis was selected as the method of data analysis.

Data analysis was initiated by the primary researcher based on repeated readings of the text and preliminary labels were given to themes that reflected key thoughts and concepts to form the bases for an initial coding scheme. A panel was formed of three individuals: (1) a PT $\mathrm{PhD}$ with expertise in health care administration, ethics, and physical therapy clinical science; (2) an OT EdD with expertise in geriatric wellness and rehabilitation, qualitative research, and OT/PT medical missions in Belize Central America; and (3) a PT ScD with expertise in clinical orthopedics, PT medical missions in rural Africa. Each panel member performed an analysis of the data individually, utilizing the de-identified transcripts based on written instructions that duplicated the analysis format used by the primary researcher.

Each of the panel member's individual coding schemes was reconciled with the initial coding scheme by that panel member and the primary 
researcher. This process of panel member and primary researcher review was then continued aggregately until there was agreement on coding scheme that was acceptable to the primary researcher and all panel members for generation of study results.

There is general agreement among qualitative researchers that concepts of credibility, transferability, dependability, confirmability, and authenticity can be used to assess the trustworthiness of qualitative research. 20,23-27 Definitions of trustworthiness assessment concepts and their sources and application of these concepts in this research study are presented in table 1.

Table 1. Tools utilized to assess research rigor

Definitions of assessment tools Application methods of assessment tools used in this study

Credibility- a reliable interpretation of Peer Examination- coding scheme was developed by collaborative data analysis of the data drawn from participants ${ }^{28}$

the primary researcher and expert panel members

Environmental Triangulation- the experiences of participants with PT care included three different communities thereby avoiding isolated phenomena

Prolonged Engagement- researcher and participants had adequate time and context exposure to: (1) understand the research context; (2) facilitate research participant frankness; and (3) recognize and avoid personal preconceptions regarding PT services in STMMM

Transferability- degree to which Detailed description of the research context was provided to enable research findings apply beyond the project ${ }^{20}$ Dependability- degree to which data and its analysis remain stable $\mathrm{e}^{20}$

Confirmability- degree to which results can be verified by others ${ }^{29}$

Authenticity- demonstration of true reporting of participant ideas ${ }^{20}$ consumers to determine the applicability to other contexts of inquiry

Digital recording and verbatim transcription of the data from interviews

Dense description of the research process to facilitate peer review of data during collaborative production of coding scheme

Reconciliation of the primary researcher's coding scheme with the independent coding schemes of expert panel members

Prolific use of supporting text for data generated themes

\section{Results}

A summary of observation themes and selected supporting text is presented in table 2 .

Table 2. Summary of research participant observations and textual support

\section{1) Need for and benefits of PT services for patients}

- $\quad$ Patients willingness to travel and wait in long lines for PT demonstrated the need for PT services (Respondent 001) "They come and they walk to get to this clinic; some parents walk up to an hour to get to the clinic. They stand in long lines to get services for their child."

- $\quad$ Activities of daily living (ADL) demands associated with life in rural Nicaragua created need for PT (Respondent 001) "Including the PT into this particular mobile medical mission trip had tremendous benefits for people.... lot of orthopedic issues...related to the life style and their working conditions."

- Patient disorders required the expertise of physical therapists for thorough diagnosis and treatment (Respondent 008) "The most challenging were pediatric patients. In particular I remember one who was a failure to develop normally who I referred to the pediatric physical therapist, a neuromuscular specialist, got some good insight back on that patient."

- Demand for PT services increased as patients attending the mobile clinic learned it was available (Respondent 005) “.... a lot of people came with a cough and a cold but when they found out PT was there they were like, "oh I have this problem." 


\section{2) Types of diagnosis and conditions seen in PT}

- The largest category of conditions reported for children and adults were orthopedic related (Respondent 003) "We saw people with neck problems, back problems, hip problems, leg problems, knee problems, ankle problems.”

- $\quad$ Additional conditions included: 1) pediatric developmental delays; 2) polio; 3) and open wounds (Multiple respondents)

\section{3) Benefits of PT services for mission team members}

- Having physical therapists enabled collaboration with and education of team members regarding the nature of PT diagnosis and the scope of feasible treatments within a mobile medical mission setting

(Respondent 002) "I thought it was a very, very beneficial collaboration between the members on the team and $P T$.

- Having PT intervention for the team members themselves was beneficial

(Respondent 004) "A number of people had musculoskeletal complaints that appeared to bother them when they were working in the stressful conditions that mobile medical missions often present with.... there was a definite need for PT intervention for the team members themselves"

- Physical therapists were able to expand their skill sets contextual to provision of PT services within the constraints of practice in these rural, limited resource environments.

(Respondent 008) "I think the people benefited and I think the physical therapists benefited from seeing how they could provide care in that kind of setting."

\section{4) Limitations of PT services in the mobile medical mission setting.}

- Language barriers in the context of an inadequate number of translators hindered the delivery of PT (Respondent 004) "Normally... there have been more than enough translators, but there were a number of personal emergencies that reduced our number of translators and slowed the process",

- $\quad$ Limited follow-up for assessment of efficacy of PT and for further intervention if necessary (Respondent 003) "Part of the issue for me would be wondering about long term follow-up. As we know from the states, we give people exercise programs, they might do it for a short period of time, then they stop, so I would assume that would be the case with people in Nicaragua"

- $\quad$ Limited availability of durable medical rehabilitation and PT treatment equipment (Respondent 004) "You simply cannot carry all the equipment that you would like to carry with you"

- $\quad$ Lack of medical provider awareness of the capability of participating physical (Respondent 002) "I think a lot of times some providers are not um, are not knowledgeable on what physical therapy and what things they can possibly do."

A summary of recommendation themes and selected supporting text is presented in Table 3.

Table 3. Summary of recommendation themes and textual support

\section{1) Recommendations to improve efficiency of delivery of PT during STMMM}

- Increase the number of translators (Respondent 004) “.... to ensure that there are an adequate number of qualified translators....is critical in these type of settings."

- Devises a more efficient means of provision of durable medical equipment for patients (Respondent 012) "Obviously you can't bring a hundred of each....so a little more, more structural supports.....other than that it's hard to say that there was any better way to of planned...." 
- $\quad$ Ensure that medical providers referring to PT are familiar with services the therapists can provide (Respondent 002) "Possibly, before the, the trip starts is to have a list of things they (physical therapists) would be able to do"

- $\quad$ Use portable computer and printing technology to provide home exercise programs more efficiently (Respondent 004) "I would look at trying to get a battery powered printer that I could hook into a laptop with that exercise program so I could tailor it to each person specifically"

- $\quad$ Conduct a STMMM that offers only PT services

(Respondent 017) "....maybe physical therapy were to be a complete clinic of their own"

2) Recommendations for additional PT treatment interventions available during STMMM

- $\quad$ Bring portable physical therapy modality devices such as ultrasound (Respondent 002) "... it might have been beneficial.... to have some type of ultrasound"

- Include physical therapy providers with women's health expertise (Respondent 002) “.... since this particular uh, mobile medical mission had some women's health providers on it, a women's health physical therapist may have been a very, beneficial”"

\section{3) Recommendations to facilitate sustainability of PT care post STMMM}

- Include Nicaraguan physical therapists in the STMMM to facilitate follow-up PT care

(Respondent 008) "....involve local physical therapy providers.... allow some referrals, some follow-up, some long term care”,

- Train healthcare workers in basic PT interventions that could in turn be taught to the community (Respondent 017) "I know on the medical side we would teach the health care providers that serve the community the things that we were doing....then that way they can educate their patient base. I think maybe PT could have benefited from that"

- $\quad$ Establish a permanent PT clinic that could provide follow-up for communities served by STMMM (Respondent 004) "Our plans for a new fulltime physical therapy clinic in the Pantasma area of Nicaragua would be a real benefit for follow-up"

\section{4) Recommendations for more comprehensive screening for patients in need of PT services}

- Utilize local missionaries to pre-screen communities for PT related problems prior to the STMMM (Respondent 007) "missionaries that were in the field could....have patients that they knew could benefit from physical therapy, have those particular people come to the clinic with a doctor's order saying this is a physical therapy patient",",

- $\quad$ Screen all patients seen during the STMMM trip for PT related problems (Respondent 018) "I would say, maybe ask the patients, after they had stated all their complaints that they wished to bring up, do you have any type of physical needs."

- Including an onsite school screening for children in need of PT services (Respondent 013) "I think it would be good if they would be able to go when the kids were in school" 


\section{Discussion}

A substantial need for health and rehabilitation services in rural Nicaragua has been described in the literature. ${ }^{7,8}$ The present study provided evidence consistent with those findings based on respondent observations of a high prevalence of ADL related etiologies of musculoskeletal disorders and the tenacity of Nicaraguans seeking PT intervention for themselves and their children. Respondents also noted the benefits of having the skill sets of physical therapists as diagnosticians and interventionists.

Respondents also reported that having a PT service on a STMMM was beneficial to the team members for both professional collaboration and for personal treatment of various orthopedic problems that arose during the trip. Similar benefits were reported by Ketter in a 1999 PT Bulletin interview of a physical therapist who had been involved in a STMMM Hurricane Mitch relief effort. ${ }^{15}$ That therapist reported that there were repeated requests for PT consults for both patients and team members.

Respondents in our study also noted that physical therapist involvement in the STMMM clinic enabled the therapists to expand their skill sets contextual to provision of PT services within the constraints of practice in these rural environments with limited resources. Similar adaptability of PT intervention and improvement of skill sets was reported in the Ketter interview. ${ }^{15}$

Another potentially useful theme that emerged from our investigation was regarding the types of diagnoses and conditions treated in the PT service. Dupre and colleagues observed that knowing the contextual health care needs of the community you're attempting to serve with medical mission outreach is of paramount importance. ${ }^{7}$ They reported that $95 \%$ of the patients their PT team encountered in rural Nicaragua presented with a chief complaint of pain and that there were limited complaints of a decline in function. Our study similarly found a high prevalence of pain related to orthopedic disorders and limited reports of dysfunction. The respondents in our study also reported other disorders including pediatric developmental delay, open wounds, posture abnormalities, and residual limitations from rare diseases such as polio. Both Dupre's and our study indicate that practitioners working in STMMM in similar environments may see a wide variety of disorders but should expect to encounter a large number of musculoskeletal disorders related to the demands of rural living in impoverished conditions.

Respondents noted a number of limitations of PT practice during this STMMM. Having only a limited number of translators was reported to slow the delivery of PT care and may have affected its quality at times. Flores assessed the impact of having a medical interpreter service for patients with Low English Proficiency (LEP) on the quality of health care in the US and found that multiple studies documented that quality of care is compromised when LEP patients do not get interpreters. $^{30}$ In the current study, patients typically had no English speaking skills, magnifying the need for a sufficient number of professional translators which was recommended by numerous respondents.

Limited means for follow-up and sustainability of PT care was also reported as a limitation of PT services during this STMMM. Jack et al. found that in stateside outpatient PT settings where follow-up is readily available, depression, poor social support, and increased pain levels during exercise were all barriers to treatment adherence. ${ }^{31}$ Sluijs et al. found that forgetfulness to do exercises, lack of positive feedback, and lack of confidence to perform the exercise correctly were all factors in patient non-adherence to PT. ${ }^{32}$ The overall degree of compliance with prescribed exercise and PT programs as reported in the literature is between one-third and two- thirds of participants . ${ }^{31-38}$ Based on these research findings, in stateside settings, it seems reasonable that the rates of compliance with PT prescription would be even less in the STMMM setting where no follow-up is available. 
Respondents recommended a number of means to address the problems of limited follow-up and sustainability. One was to involve local PT providers in the STMMM and patient follow-up at local facilities. This would be feasible if Mission Para Cristo is able to establish a PT clinic in the region. It was also recommended that physical therapists on the STMMM team teach local health providers how to instruct patients in PT related group classes. In 2014, Mission Para Cristo included simple PT interventions in the continuing education for its Community Health Educators. This allowed the Community Health Educators to provide basic PT services for their patients during community health encounters.

Limitations in the amount of durable medical and treatment equipment that can be taken on a STMMM trip was a topic of respondent discussion. Information regarding the types of diagnoses encountered in a particular setting, as gathered in this study, or in the needs assessment study by Dupree, could be helpful in determining the type of physical therapy and durable medical equipment to pack. ${ }^{7}$ However, as one respondent in this study noted, the need is ultimately determined by which patients happen to come to that particular STMMM clinic.

Another noteworthy limitation to the delivery of PT during this STMMM was the lack of medical provider awareness of physical therapists' scope of practice. This could be a significant problem when using a medical referral model as it constrains referrals to the referring medical provider's familiarity of PT practice. Stanton et al. found a deficit in resident physicians' knowledge of PT. ${ }^{39}$ Uili et al. found that physician knowledge of PT procedures was dependent on medical specialty and duration of practice. $^{40}$ Harrison and Kisicki assessed the knowledge of Arizona-based physician assistants (PAs) and nurse practitioners (NPs) regarding technical and professional skill sets of physical therapists. ${ }^{41}$ While the knowledge level of PAs was greater than NPs, both demonstrated notable limitations in the understanding of basic modalities and professional interventions.

One respondent offered a simple solution to the problem of limited knowledge of PT by medical providers, which was to familiarize them with the expertise and skill sets of the physical therapists participating in the STMMM prior to starting clinic. An additional option would be to use a direct access model with the inclusion of a physical therapist in the triage process and, thereby, ensure that all patients with PT related diagnosis are seen by a PT at some point in their clinic encounter.

Respondent recommendations to increase community exposure to PT within the STMMM model had strengths and weaknesses. One recommendation was to conduct a clinic that exclusively provided PT services. While a dedicated PT clinic would likely increase community access to PT, it would not provide a venue for treatment of those patients found to have a medical problem. Another recommendation was to carry out a community screening program for problems contextual to PT prior to the STMMM using resident missionaries. A better option for this type of screening program would be to utilize government recognized Health Educators employed by Mission Para Cristo, who already have a basic knowledge of general health issues and practices. Another respondent recommendation was to screen all patients seen during the STMMM clinic for PT related issues. As previously noted, this could be facilitated by having a physical therapist work with the triage team. A respondent also recommended that the STMMM be used to facilitate onsite PT screening and treatment of children while at school. While this is likely feasible and worthwhile, it would not facilitate developmental screening beginning at age nine months as recommended by the American Academy of Pediatrics (AAP). ${ }^{42}$

There were a number of constraints in this study. Phone interview was the most feasible means of ensuring timely respondent contact due to medical professional and nursing student travel schedules and obligations. Phone interviews limit 
respondents' time to think about their answers to the interview questions. This limitation was hopefully negated by providing participants with a copy of the interview questions at the time of informed consent. The use of a phone interview also has the potential to introduce researcher interpretation bias unless exacting measures are taken to ensure data dependability. Steps taken to ensure data and analysis dependability included: (1) verbatim transcription of the digitally recorded text to avoid inadvertent interpolation of content; (2) close adherence to the text during production of the primary researcher's initial coding scheme; (3) scrutiny of the primary researcher's initial coding scheme by subjecting it to reconciliation with the coding drafts of a three member panel from diverse professional backgrounds; (4) support of analysis themes with multiple textual excerpts.

Another limitation of this study was the exclusion of STMMM participants that did not have U.S. based phone service. This eliminated Nicaraguan translators and transporters participation in the study, both of whom could have provided a unique prospective regarding the inclusion of PT services in the STMMM trip. Since the completion of this research, a no cost phone service to the US has been installed at Mission Para Cristo. This could facilitate the inclusion of the Nicaraguan members of STMMM in the future or another means of more inclusive data collection could be prearranged in harmony with the STMMM planning.

Not having the input of local health care providers, particularly physical therapists, in the study was an intrinsic limitation. The lack of physical therapists and other community based health care providers in the areas served by these efforts is of course part of the rationale for conducting STMMM in the region. There are future plans to add a fulltime Nicaraguan physical therapist to the staff of Mission Para Cristo. That individual's participation could facilitate follow-up PT care, provide local provider insight into future qualitative research, and provide a means to collect post STMMM outcomes data to facilitate quantitative inquires.

\section{Conclusion}

This study examined the acumens of medical mission participants regarding the inclusion of physical therapists in a STMMM team working in rural Nicaragua. Four major themes emerged contextual to the research question, "What are the observations of medical mission team members regarding the inclusion of PT services on a mobile medical mission team to underserved areas of Nicaragua?" These included need for and benefits of PT for patients, identification of types of diagnosis and conditions seen in PT, benefits of PT services for mission team members, and limitations of PT services in the mobile medical mission setting.

There were also four major themes that emerged contextual to the research question, "What are the recommendations of team members for improvement in the delivery of PT services during a mobile medical mission to underserved areas of Nicaragua?" These included recommendations to improve efficiency of delivery of PT, recommendations to make additional PT treatment interventions available, recommendations to facilitate follow-up assessment, and sustainability of PT care and recommendations for more comprehensive screening for patients in need of PT intervention.

The results were consistent with the objective of the study, which was to add to the understanding of corollaries associated with the addition of PT services to a STMMM and to discern measures that could improve the delivery of PT services in that context. Additional research is needed to further assess the utility and clinical efficacy of having PT services in STMMM. Additionally, mechanisms need to be put in place to facilitate outcomes data gathering for quantitative research to determine best practices for PT delivered in the STMMM and to discern potentially undesirable consequences of 
delivery of PT in that context as has been identified with other short term medical services. ${ }^{43,44}$

\section{References}

1. Peters DH, Garg A, Bloom G, Walker DG, Brieger WR, Rahman MH. Poverty and access to health care in developing countries. Ann N Y Acad Sci. 2008;1136:16171.

http://dx.doi.org/10.1111/j.1749-6632.1970.tb39320.x

2. Campbell J, Dussault G, Buchan J, Pozo-Martin F, Guerra Arias M, Leone C, et al. A universal truth: no health without a workforce [Internet]. Global Health Workforce Alliance and World Health Organization. 2013 Nov; [cited 2014 Jan 5] Available from:

http://www.who.int/workforcealliance/knowledge/resour ces/hrhreport2013/en/

3. Officer A, Posarac A. World report on disability [Internet]. World Health Organization. Available from: http://whqlibdoc.who.int/publications/2011/9789240685 215_eng.pdf [Published 2011] [cited 2013 March 15]

4. Liao H, Lai J, Chai H, Yaung C, Liao W. Supply of physical therapists in member countries of the World Confederation for Physical Therapy. Physiotherapy Theory and Practice. 1997;13:227-34. http://dx.doi.org/10.3109/09593989709036466

5. Gupta N, Castillo-Laborde C, Landry MD. Healthrelated rehabilitation services: assessing the global supply of and need for human resources. BMC Health Serv Res. 2011;11:276-87.

http://dx.doi.org/10.1186/1472-6963-11-276

6. World Health Organization. Nicaragua: country cooperation strategy at a glance [Internet]. https://extranet.who.int/iris/restricted/bitstream/10665/13 6781/1/ccsbrief_nic_es.pdf

[cited 2014 Apr 28] [Currently only available in Spanish]

7. Dupre AM, Goodgold S. Implementation of a physical therapy needs assessment in Nicaragua [abstract]

[Internet]. Physical Therapy Online. 2005. http://aptaapps.apta.org/Abstracts/abstract.aspx?abnum= QUNQUFBUMDV8UE8tU0ktMTMtVEguSFRNTA Published 2005. [cited 2014 Apr 28]

8. Cano O. Interview [Internet]. 2003 February. [Cited by Jarquin FT. International Disability Monitor. Regional Report of the Americas (Nicaragua)]. 2004 [cited 2014 May 8] Available from: http://www.idrmnet.org/content.cfm?id=5E5A75\&m=3
9. Spring of hope Uganda [Internet]. [cited 2014 Apr 29] Available from:

http://www.springofhope.org.uk/index.html

10. Global health outreach [Internet]. [cited 2014 Apr 29] Available from: http://www.cpti.org/mis_opps.html

11. Health Volunteers Overseas [Internet]. Physical Therapy. [cited 2014 Apr 29] Available from: http://www.hvousa.org/ourwork/programs/physicaltherapy/

12. Short term missions.com [Internet]. [cited 2014 Apr 29] Available from:

http://www.shorttermmissions.com/trips/8117/?STM=09 6bb91a9b4b2cce37c3070c193996d6

13. Standing with Hope [Internet]. [cited 2015 Jan 7] Available from:

http://www.standingwithhope.com/outreaches/prosthetic s/participate-trip/

14. Bajkiewicz C. Evaluating short-term missions: how can we improve? J Christ Nurs. 2009;26:110-14.

15. Ketter P. PT helps Nicaraguans recover from hurricane. PT Bulletin. 1999;14:3.

16. Nixon S, Cleaver S, Stevens M, Hard J, Landry M. The role of physical therapists in natural disasters: what can we learn from the earthquake in Haiti? Physiother Can. 2010;62:167-8. Available from:

http://dx.doi.org/10.3138/physio.62.3.167

17. Faanes E. Experiences of physical therapists who participate in disaster relief work in Haiti [Doctor of Physical Therapy Research Paper]. Saint Paul, MN: St. Catherine University Sophia. 2012. [cited 2013 March 20] Available from:

http://sophia.stkate.edu/cgi/viewcontent.cgi?article=1013 \&context=dpt_papers

18. Sandelowski M. Whatever happened to qualitative description? Res Nurs Health. 2000;23:334-40. http://dx.doi.org/10.1002/1098240x(200008)23:4\%3C334::aid-nur9\%3E3.0.co;2-g

19. Sequeira M, Espinoza H, Amador JJ, Domingo G, Quintanilla M, de los Santos T. The Nicaraguan health system [Internet]. Seattle, Washington: PATH; 2011. [cited 2014 June 3] Available from: http://www.path.org/publications/files/TS-nicaraguahealth-system-rpt.pdf 
20. Nicholls D. Qualitative research: part three-methods. Int J Ther Rehabil. 2009;6:638-47. Available from: http://dx.doi.org/10.12968/ijtr.2009.16.12.45433

21. McLellen E, Macqueen K, Neidig J. Beyond the qualitative interview: data preparation and transcription. Field Methods. 2003;15:63-84. Available from: http://dx.doi.org/10.1177/1525822x02239573

22. Hsiu-Fang H, Shannon S. Three approaches to qualitative content analysis. Qual Health Res 2005;15:1277-88. Available from: http://dx.doi.org/10.1177/1049732305276687

23. Mays N, Pope C. Rigour and qualitative research. BMJ. 1995;311(6997):109-12. Available from: http://dx.doi.org/10.1136/bmj.311.6997.109

24. Koch T. Establishing rigour in qualitative research: the decision trail. J Adv Nurs. 1993;19: 976-86. Available from: http://dx.doi.org/10.1111/j.13652648.1994.tb01177.x

25. Seale C, Silverman D. Ensuring rigour in qualitative research. European J Pub Heal. 1997;7:379-84. Available from: http://dx.doi.org/10.1093/eurpub/7.4.379

26. Golafshani N. Understanding reliability and validity in qualitative research. The Qualitative Report. 2003;8:597-607. [cited 2014 May 30] Available from: http://www.nova.edu/ssss/QR/QR8-4/golafshani.pdf

27. Tobin GA, Begley CM. Methodological rigour within a qualitative framework $J A d v$ Nurs. 2004;48:38896.

28. Lincoln YS, Guba EG. Naturalistic inquiry. Newbury Park, CA: Sage Publications; 1985.

29. Trochim MK. Qualitative validity [Internet]. [revised 2006 Oct 20] [cited 2014 June 3] Available from:

http://www.socialresearchmethods.net/kb/qualval.php

30. Flores G. The impact of medical interpreter services on the quality of health care: a systematic review Med Care Res Rev 2005;62;255-99. Available from: http://dx.doi.org/ 10.1177/1077558705275416

31. Jack K, McLeanb SM, Moffett JK, Gardiner E. Barriers to treatment adherence in physiotherapy outpatient clinics: a systematic review. Man Ther. 2010;15: 220-8. Available from: http://dx.doi.org/10.1016/j.math.2009.12.004 [Epub 2010 Feb 16]
32. Sluijs EM, Kok GJ, van der Zee J. Correlates of exercise compliance in physical therapy. Physical Therapy. 1993;73:771-82.

33. Picorelli AM, Pereira DS, Felício DC, Dos Anjos DM, Pereira DA, Assis MG, et al. Adherence of older women with strength training and aerobic exercise. Clin Interv Aging. 2014;9:323-31. Available from: http://dx.doi.org/10.2147/CIA.S54644

34. Medina-Mirapeix F, Escolar-Reina P, GascónCánovas JJ, Montilla-Herrador J, Jimeno-Serrano FJ, Collins SM. Predictive factors of adherence to frequency and duration components in home exercise programs for neck and low back pain: an observational study. $B M C$ Musculoskelet Disord. 2009;10:155. Available from: http://dx.doi.org/10.1186/1471-2474-10-155.

35. Härkäpää K, Järvikoski A, Mellin G, Hurri H. A controlled study on the outcome of inpatient and outpatient treatment of low back pain. Scand J Rehabil Med. 1989;21:81-9.

36. Ice R. Long-term compliance. Phys Ther. 1985;65:1832-9.

37. Martin JE, Dubbert PM. Exercise applications and promotion in behavioral medicine: current status and future directions. J Consult Clin Psychol. 1982;50:100417.

38. Jette AM. Improving patient cooperation with arthritis treatment regimens. Arthritis

Rheum.1982;25:447-53.

39. Stanton PE, Fox FK, Frangos KM, Hoover DH, Spilecki GM. Assessment of resident physicians' knowledge of physical therapy. Phys Ther. 1985;65:2730 .

40. Uili RM, Shepard KF, Savinar E. Physician knowledge and utilization of physical therapy procedures Phys Ther. 1984;64:1523-30.

41. Harrison $\mathrm{FG}^{1}$, Kisicki DS. How knowledgeable are physician assistants and nurse practitioners about physical therapy? J Allied Health. 2006;35(3):e227-339.

42. Grissom M. Disorders of childhood growth and development: screening and evaluation of the child who misses developmental milestones. FP Essent. 2013;410:32-44.

43. Montgomery, L. Short-term medical missions: enhancing or eroding health? 
Missiology.1993;21(3):333-41. Available from: http://dx.doi.org/10.1177/009182969302100305

44. Sykes K. Short-term medical service trips: a systematic review of the evidence. Am J Public Heal. 2014;104:e38-48. Available from: http://dx.doi.org/10.2105/AJPH.2014.301983. [Epub 2014 May 15].

\section{Appendix: A (Interview Questions)}

1) Did you have any significant interaction with physical therapy services during the mobile medical mission trip? For instance, did you work directly with the physical therapy service, refer patients to the physical therapy service or assist in the coordination of the service in some way?(If the answer is no, the participant will be thanked for their participation and informed that this is the conclusion of their interview).
2) Tell us about your experiences with physical therapy service during this mission trip?

3) What were the most common medical diagnoses of the patients you referred to physical therapy? What were the most interesting or challenging diagnoses of patients you referred to physical therapy? (Medical Providers)

4) What were the most common physical therapy diagnoses of patients you treated in physical therapy? What were the most interesting or challenging diagnoses of patients you treated in therapy? (Physical Therapy Providers)

5) What were the benefits and limitations associated with including physical therapy services in the mobile medical mission context?

6) What changes could be made to improve the delivery of physical therapy care in the mobile medical mission context?

\section{Peer Reviewed}

Competing Interests: None declared.

Correspondence: Douglas Steele, Harding University, United States. dasteele@harding.edu ; Candice Beitman, University of Indianapolis, United States. cbeitman@uindy.edu

Cite this article as: Steele D, Beitman C. Inclusion of physical therapy services on a short term mobile medical mission team to Nicaragua: a qualitative description study of team members' observations and recommendations for improvement. Christian Journal for Global Health (Nov 2015), 2(2): 39-51.

(C) Steele D, Beitman C This is an open-access article distributed under the terms of the Creative Commons Attribution License, which permits unrestricted use, distribution, and reproduction in any medium, provided the original author and source are properly cited. To view a copy of the license, visit http://creativecommons.org/licenses/by/3.0/

www.cjgh.org 